\title{
SYSTEMS OF DIFFERENTIAL EQUATIONS SUBJECT TO MILD INTEGRAL CONDITIONS
}

\author{
WILLIAM F. TRENCH
}

\begin{abstract}
It is shown that solutions of a system $x^{\prime}=f(t, x)$ approach constant vectors as $t \rightarrow \infty$, under assumptions which do not require that $\|f(t, x)\| \leqslant$ $w(t,\|x\|)$, where $w$ is nondecreasing in $\|x\|$, and which permit some or all of the integral smallness conditions on $f$ to be stated in terms of ordinary-rather than absolute-convergence. Estimates of the order of convergence are given.
\end{abstract}

Theorems which imply that solutions of a system

$$
x^{\prime}=f(t, x)
$$

approach constant vectors as $t \rightarrow \infty$ are usually obtained by assuming that

$$
\|f(t, x)\| \leqslant w(t,\|x\|),
$$

where $w$ is decreasing in $r$ for $r \geqslant 0$, and subject to some integral condition such as

$$
\int^{\infty} w(t, r) d t<\infty, \quad r \geqslant 0
$$

for examples, see $[1,4,6]$. Sometimes, instead of $(3)$, it is required that the equation $r^{\prime}=w(t, r)$ have a bounded positive solution on some half-line $\left[t_{0}, \infty\right)$; for example, see [2]. Clearly, any integral condition like (3) requires absolute convergence, and there are systems for which no majorizing function $w$ as in (2) is increasing in $\|x\|$. Here we present results which do not require this kind of bound on $f$, and are based on integral conditions involving ordinary - that is, not necessarily absolute-convergence of some of the improper integrals in question. Moreover, our results include information on the order of convergence of solutions of (1).

Throughout this paper the norm \|\| of a vector or matrix is the sum of the absolute values of its elements, and $W$ and $K$ are as in the following assumption. The motivation for this definition will become clear after the statement of the main theorem (Remark 1, below).

Assumption A. The $n \times n$ matrix function $W$ is continuously differentiable and invertible on $[\bar{T}, \infty)$ for some $\bar{T} \geqslant 0$, and

$$
\int_{t}^{\infty}\left\|W(t)\left(W^{-1}(s)\right)^{\prime}\right\| d s \leqslant K<\infty, \quad \cdot \quad t \geqslant \bar{T} .
$$

Received by the editors January 18, 1982 and, in revised form. April 12, 1982: presented to the Society, March 17, 1982.

1980 Mathematics Subject Classification. Primary 34C11, 34D10.

if 1983 American Mathematical Society $0002-9939 / 82 / 0000-0866 / \$ 02.25$ 
Henceforth we assume that $t \geqslant \bar{T}$. Notice that

$$
\int^{\infty}\left\|\left(W^{-1}(s)\right)^{\prime}\right\| d s<\infty
$$

and so

$$
\lim _{t \rightarrow \infty} W^{-1}(t) \text { exists (finite) }
$$

because of (4).

LEMMA 1. If $q$ is a continuous n-vector function on $[T, \infty)$ and $\int^{\infty} W(t) q(t) d t$ converges, then $\int^{\infty} q(t) d t$ converges, and

$$
\left\|W(t) \int_{t}^{\infty} q(s) d s\right\| \leqslant(1+K) \rho(t), \quad t \geqslant T,
$$

where

$$
\rho(t)=\sup _{\lambda \geq t}\left\|\int_{\lambda}^{\infty} W(s) q(s) d s\right\| .
$$

Proof. With

$$
p(t)=\int_{t}^{\infty} W(s) q(s) d s
$$

integration by parts yields

$$
\int_{t}^{\bar{t}} q(s) d s=\int_{t}^{\bar{t}} W^{-1}(s) W(s) q(s) d s=-\left.W^{-1}(s) p(s)\right|_{t} ^{i}+\int_{t}^{\bar{t}}\left(W^{-1}(s)\right)^{\prime} p(s) d s .
$$

From (6), (8), and (9),

$$
\left\|\left(W^{-1}(s)\right)^{\prime} p(s)\right\| \leqslant \rho(T)\left\|\left(W^{-1}(s)\right)^{\prime}\right\|, \quad s \geqslant T,
$$

and $\lim _{t \rightarrow \infty}^{-} W^{-1}(\bar{t}) p(\bar{t})=0$; hence, because of (5), we can let $\bar{t} \rightarrow \infty$ in (10) and obtain

$$
\int_{t}^{\infty} q(s) d s=W^{-1}(t) p(t)+\int_{t}^{\infty}\left(W^{-1}(s)\right)^{\prime} p(s) d s .
$$

Multiplying by $W(t)$ and invoking (4), (8), and (9) yields (7).

Definition 1. For $T \geqslant 0$, let $H(T)$ be the Banach space of continuous $n$-vector functions $h$ on $[T, \infty)$ such that $W h$ is bounded, with the norm

$$
N(T ; h)=\sup _{t \geqslant T}\|W(t) h(t)\| .
$$

If $M>0$, let

$$
H_{M}(T)=\{h \in H(T) \mid N(T ; h) \leqslant M\} .
$$

The following is our main theorem:

THEOREM 1. For a given vector $c$, suppose there are constants $M>0$ and $T_{0} \geqslant 0$ such that $f$ is continuous on

$$
\Omega=\left\{(t, x) \mid t \geqslant T_{0},\|W(t)(x-c)\| \leqslant M\right\}
$$


and the integral

$$
J(t ; h)=\int_{t}^{\infty} W(s) f(s, c+h(s)) d s, \quad t \geqslant T,
$$

converges if $h \in H_{M}(T)$ and $T \geqslant T_{0}$. Suppose also that

$$
\left\|J\left(t ; h_{1}\right)-J\left(t ; h_{2}\right)\right\| \leqslant \delta N\left(T ; h_{1}-h_{2}\right), \quad t \geqslant T,
$$

whenever

$$
h_{1}, h_{2} \in H_{M}(T) \text { with } T \geqslant T_{0} \text {, }
$$

where

$$
0<\delta<1 /(1+K) \text {. }
$$

Then (1) has a solution $x_{0}$ which is defined for $t$ sufficiently large and satisfies

$$
\lim _{t \rightarrow \infty} W(t)\left(x_{0}(t)-c\right)=0 \text {; }
$$

moreover, if $x_{1}$ is any solution of (1) such that

$$
\lim _{t \rightarrow \infty} W(t)\left(x_{1}(t)-c\right)=0
$$

then

$$
x_{1}(t)=x_{2}(t)
$$

for $t$ sufficiently large.

REMARK 1. In our examples, $W=\operatorname{diag}\left[w_{1}, \ldots, w_{n}\right]$ with $w_{i}>0$ and $w_{i}^{\prime} \geqslant 0$, in which case (4) holds with $K=n$, and (17) implies that the components of $x_{0}$ approach $c$ at possibly different rates. We state and prove our theorems under the more general Assumption A because this does not complicate the proofs and the more general formulation may be useful in some applications.

Proof of Theorem 1. If $h \in H_{M}(T)$ with $T \geqslant T_{0}$, then

$$
\|J(t ; h)\| \leqslant\|J(t ; h)-J(t ; 0)\|+\|J(t ; 0)\| \leqslant \delta M+\|J(t ; 0)\|,
$$

from (14) with $h_{1}=h$ and $h_{2}=0$. Now choose $T_{1} \geqslant T_{0}$ so that

$$
\delta M+\sup _{t \geqslant T_{1}}\|J(t ; 0)\| \leqslant M /(1+K)
$$

which is possible because of (16) and the convergence of $J(t ; 0)=\int_{t}^{\infty} W(s) f(s, c) d s$. From (20) and (21),

$$
\|J(t ; h)\| \leqslant M /(1+K) \quad \text { if } t \geqslant T_{1} \text { and } h \in B_{M}\left(T_{1}\right) .
$$

If $h \in H_{M}\left(T_{1}\right)$, define $\hat{h}=\sigma h$ by

$$
\hat{h}(t)=-\int_{t}^{\infty} f(s, c+h(s)) d s, \quad t \geqslant T_{1} .
$$

From (13) and Lemma 1 with $q(t)=f(t, c+h(t)), \hat{h}$ is defined and satisfies the inequality

$$
\|W(t) \hat{h}(t)\| \leqslant(1+K) \sup _{\lambda \geqslant t}\|J(t ; h)\|, \quad t \geqslant T_{1} .
$$

From this and (22), $\|W(t) \hat{h}(t)\| \leqslant M, t \geqslant T_{1}$. Therefore, $\hat{h} \in H_{M}\left(T_{1}\right)$; that is, $\mathcal{T}$ transforms $H_{M}\left(T_{1}\right)$ into itself. 
Now suppose $h_{1} \in B_{M}\left(T_{1}\right)$ and $\hat{h}_{i}=\Im h_{i}(i=1,2)$. Then Lemma 1 with

$$
g(t)=f\left(t, c+h_{1}(t)\right)-f\left(t, c+h_{2}(t)\right)
$$

implies that

$$
\left\|W(t)\left(\hat{h}_{1}(t)-\hat{h}_{2}(t)\right)\right\| \leqslant(1+K)\left\|J\left(t ; h_{1}\right)-J\left(t ; h_{2}\right)\right\|, \quad t \geqslant T_{1},
$$

and so, from (11) and (14) (with $T=T_{1}$ ),

$$
N\left(T_{1} ; \hat{h}_{1}-\hat{h}_{2}\right) \leqslant \delta(1+K) N\left(T_{1} ; h_{1}-h_{2}\right) .
$$

Hence, from (16), $: T$ is a contraction mapping of $H_{M}\left(T_{1}\right)$ into itself, and therefore there is an $h_{0}$ in $H_{M}\left(T_{1}\right)$ such that $h_{0}=\widetilde{T} h_{0}$; that is,

$$
h_{0}(t)=-\int_{t}^{\infty} f\left(s, c+h_{0}(s)\right) d s, \quad t \geqslant T_{1} .
$$

From Lemma 1 with $q(t)=f\left(t, c+h_{0}(t)\right), \lim _{t \rightarrow \infty} W(t) h_{0}(t)=0$. Therefore, the function $x_{0}=c+h_{0}$ satisfies (1) and (17).

If $x_{1}$ satisfies (1) and (18), then $h_{1}=x_{1}-c$ is in $H_{M}\left(T_{2}\right)$ for some $T_{2} \geqslant T_{1}$, and

$$
h_{1}(t)-h_{0}(t)=\int_{t}^{\infty}\left[f\left(s, c+h_{0}(s)\right)-f\left(s, c+h_{1}(s)\right)\right] d s, \quad t \geqslant T_{2} .
$$

By an argument like that which led to (23),

$$
N\left(T_{2} ; h_{1}-h_{0}\right) \leqslant \delta(1+K) N\left(T_{2} ; h_{1}-h_{0}\right),
$$

which implies that $h_{1}(t)=h_{0}(t)$ for $t \geqslant T_{2}$, because of (16). This implies (19) for $t \geqslant T_{2}$, and completes the proof.

We now apply Theorem 1 to the system

$$
x^{\prime}=A(t) \psi(x)+g(t), \quad t>0 .
$$

First we need the following definition:

Definition 2. A vector $c$ is a Lipschitz point of a vector function $\psi$ if there are constants $\rho, \lambda>0$ such that $\psi(x)$ is defined whenever

$$
\|x-c\| \leqslant \rho
$$

and

$$
\left\|\psi\left(x_{1}\right)-\psi\left(x_{2}\right)\right\| \leqslant \lambda\left\|x_{1}-x_{2}\right\|
$$

if $\left\|x_{i}-c\right\| \leqslant \rho, i=1,2$.

THEOREM 2. Suppose $A$ is an $n \times m$ matrix function and $g$ is an $n$-vector function, both continuous on $[0, \infty)$, and $c$ is a Lipschitz point of the m-vector function $\psi$. Suppose also that

$$
\int^{\infty} W(t)[A(t) \psi(c)+g(t)] d t
$$

converges and

$$
\int^{\infty}\|W(t) A(t)\|\left\|W^{-1}(t)\right\| d t<\infty
$$

Then the conclusions of Theorem 1 hold for (24). 
Proof. Let

$$
\sigma=\sup _{t \geq \bar{T}}\left\|W^{-1}(t)\right\|,
$$

which is finite because of (6). Let $\delta$ be any number that satisfies (16), let $\lambda$ be as in (26), and choose $T_{0}$ so that

$$
\int_{T_{0}}^{\infty}\|W(s) A(s)\|\left\|W^{-1}(s)\right\| d s \leqslant \delta / \lambda,
$$

which is possible because of (28). Henceforth, let $t \geqslant T_{0}$. Finally, let

$$
M=\rho / \sigma,
$$

with $\rho$ as in (25). We will show that $T_{0}$ and $M$ satisfy the requirements of Theorem 1 , for

$$
f(t, x)=A(t) \psi(x)+g(t) .
$$

We must first show that $f$ is continuous on $\Omega$ as defined in (12). If $(t, x) \in \Omega$, then

$$
\|x-c\| \leqslant\left\|W^{-1}(t)\right\|\|W(t)(x-c)\| \leqslant \sigma M=\rho,
$$

because of (29) and (31). Since $\psi$ is continuous for all $x$ satisfying (25), while $A$ and $g$ are continuous on $[0, \infty)$, it follows that $f$ is continuous on $\Omega$, and $f(t, c+h(t))$ is continuous on $[T, \infty)$ if $h \in H_{M}(T)$ with $T \geqslant T_{0}$. Moreover, if (15) holds, then

$$
\begin{aligned}
\left\|\psi\left(c+h_{1}(t)\right)-\psi\left(c+h_{2}(t)\right)\right\| & \leqslant \lambda\left\|h_{1}(t)-h_{2}(t)\right\| \\
& \leqslant \lambda\left\|W^{-1}(t)\right\|\left\|W(t)\left(h_{1}(t)-h_{2}(t)\right)\right\| \\
& \leqslant \lambda\left\|W^{-1}(t)\right\| N\left(T ; h_{1}-h_{2}\right), \quad t \geqslant T .
\end{aligned}
$$

where we have used (11) and (26). This and (30) imply that

$$
\left\|\int_{t}^{\infty} W(s) A(s)\left[\psi\left(c+h_{1}(s)\right)-\psi\left(c+h_{2}(s)\right)\right] d s\right\| \leqslant \delta N\left(T ; h_{1}-h_{2}\right),
$$$$
t \geqslant T \text {. }
$$

With $f$ as in (32), the functional $J$ in (13) becomes

$$
J(t ; h)=\int_{t}^{\infty} W(s)[A(s) \psi(c+h(s))+g(s)] d s .
$$

From the convergence of $(27), J(t ; 0)$ exists. This and the convergence of the integral in (33) with $h_{1}=h$ and $h_{2}=0$ imply that $J(t ; h)$ exists for all $h \in H_{M}(T)$, if $t \geqslant T \geqslant T_{0}$. Knowing this, we can conclude from (33) that (14) holds whenever (15) does. This completes the proof of Theorem 2.

Stronger results are available for a linear system

$$
x^{\prime}=A(t) x+g(t), \quad t>0 .
$$

We omit the proof of the following theorem, which is similar to that of Theorem 2.

THEOREM 3. Suppose the $n \times n$ matrix function $A$ and the $n$-vector function $g$ are continuous on $[0, \infty)$,

$$
\int^{\infty}\left\|W(t) A(t) W^{-1}(t)\right\| d t<\infty
$$


and

$$
\int^{\infty} W(t)[A(t) c+g(t)] d t
$$

converges for a given constant vector $c$. Then (34) has a unique solution $x_{0}$ which satisfies (17).

The next theorem follows easily from this and elementary properties of linear systems.

THEOREM 4. Suppose $A$ and $g$ are continuous on $[0, \infty)$, (35) holds, and $\int^{\infty} W(t) A(t) d t$ and $\int^{\infty} W(t) g(t) d t$ converge. Then (34) has a unique solution which satisfies (17) for any given constant vector c, and every solution of (34) satisfies (17) for some $c$.

Theorem 4 with $W=I$ contains a result of Brauer [1, Lemma 2], who assumed that $\int^{\infty}\|A(t)\| d t<\infty$ and $\int^{\infty}\|g(t)\| d t<\infty$.

In the following examples, $c=\operatorname{col}\left[\alpha_{1}, \alpha_{2}\right]$ and $x_{1}$ and $x_{2}$ are the components of a solution vector $x$.

EXAMPLE 1. The system

$$
\left[\begin{array}{l}
x_{1}^{\prime} \\
x_{2}^{\prime}
\end{array}\right]=\frac{4 \sin t}{t^{4}\left(x_{1}-x_{2}\right)^{2}}\left[\begin{array}{ccc}
1 & 2 & -t \\
t^{2} & t & -1
\end{array}\right]\left[\begin{array}{c}
x_{1}^{2} \\
x_{1} x_{2} \\
x_{2}^{2}
\end{array}\right]+\frac{\sin t}{t^{3}}\left[\begin{array}{c}
1 \\
1-t
\end{array}\right], \quad t>0
$$

is of the form (24). If $W=\operatorname{diag}\left[t^{q}, t^{q-1}\right]$ with $q \geqslant 1$, then (28) holds. If $\alpha_{1} \neq \alpha_{2}$, then $c$ is a Lipschitz point of $\psi$ in (37), and (27) converges if $q<3$. Therefore, Theorem 2 implies that (37) has a solution such that

$$
x_{1}(t)=\alpha_{1}+o\left(t^{-q}\right), \quad x_{2}(t)=\alpha_{2}+o\left(t^{-q+1}\right)
$$

for all $q<3$, provided $\alpha_{1} \neq \alpha_{2}$. If $\alpha_{1}=-\alpha_{2} \neq 0$, then (27) converges for $q<4$, and so (38) holds for all $q<4$.

EXAMPLE 2. For the linear system

$$
\left[\begin{array}{l}
x_{1}^{\prime} \\
x_{2}^{\prime}
\end{array}\right]=\left[\begin{array}{cc}
2 t^{-2} \sin t & 3 t^{-1} \\
t^{-3} & t^{-3} \sin t
\end{array}\right]\left[\begin{array}{l}
x_{1} \\
x_{2}
\end{array}\right]+\left[\begin{array}{c}
6 t^{-1} \\
-2 t^{-3}
\end{array}\right],
$$

(35) holds if $W=\operatorname{diag}\left[t^{q}, t^{q+1}\right]$ with $q \geqslant 0$, and (36) converges if $\alpha_{1}$ is arbitrary, $\alpha_{2}=-2$, and $q<1$. Hence, from Theorem 3, (39) has a solution such that

$$
x_{1}(t)=\alpha_{1}+o\left(t^{-q}\right), \quad x_{2}(t)=-2+o\left(t^{-q^{-1}}\right)
$$

for all $q<1$. If $\alpha_{1}=-\alpha_{2}=2$, then (36) converges if $q<2$, and so (40) holds for all $q<2$ if $\alpha_{1}=2$.

EXAMPLE 3. We now exhibit a system $x^{\prime}=A(t) x$ whose solutions all tend rapidly to constant vectors, even though $\int^{\infty}\|A(t)\| d t=\infty$. To this end, we first observe that $\int^{\infty} t^{\nu} e^{\mu t} \sin \left(e^{t}\right) d t$ converges for all $\nu$ if $\mu<1$; if $\mu=0$, it converges conditionally if $\nu \geqslant-1$, absolutely if $\nu<-1$. (To see this, substitute $\tau=e^{t}$.) Now consider the system

$$
\left[\begin{array}{l}
x_{1}^{\prime} \\
x_{2}^{\prime}
\end{array}\right]=\sin \left(e^{t}\right)\left[\begin{array}{ll}
a t^{-3 / 2} & b t^{-1 / 2} \\
c t^{-5 / 2} & d t^{-3 / 2}
\end{array}\right]\left[\begin{array}{l}
x_{1} \\
x_{2}
\end{array}\right]
$$


where $a, b, c, d$ are constants and $b \neq 0$, so that $\int^{\infty}\|A(t)\| d t=\infty$, and let $W=$ $\operatorname{diag}\left[e^{\mu t}, t e^{\mu t}\right]$ with $0 \leqslant \mu<1$. Here $\int^{\infty} W(t) A(t) d t$ converges and (35) holds; hence, Theorem 4 implies that if $\alpha_{1}$ and $\alpha_{2}$ are arbitrary, then (41) has a solution such that

$$
x_{1}(t)=\alpha_{1}+o\left(e^{-\mu t}\right) . \quad x_{2}=\alpha_{2}+o\left(t^{-1} e^{-\mu t}\right)
$$

for all $\mu<1$.

EXAMPLE 4. Suppose $a, b, f \in C(0, \infty)$ and $z_{1}$ and $z_{2}$ are solutions of

$$
z^{\prime \prime}+a(t) z=0 . \quad t>0 .
$$

with wronskian $\omega \neq 0$. Then $y$ satisfies

$$
y^{\prime \prime}+a(t) y=b(t) y+f(t) . \quad t>0 .
$$

if and only if

$$
y^{(r)}=x_{1} z_{1}^{(r)}+x_{2} z_{2}^{(r)} . \quad r=0.1
$$

where

$$
\left[\begin{array}{l}
x_{1}^{\prime} \\
x_{2}^{\prime}
\end{array}\right]=\frac{b}{\omega}\left[\begin{array}{cc}
-z_{1} z_{2} & -z_{2}^{2} \\
z_{1}^{2} & z_{1} z_{2}
\end{array}\right]\left[\begin{array}{l}
x_{1} \\
x_{2}
\end{array}\right]+\frac{f}{\omega}\left[\begin{array}{c}
-z_{2} \\
z_{1}
\end{array}\right] .
$$

Let $\alpha_{1}$ and $\alpha_{2}$ be given constants, define $g=b\left(\alpha_{1} z_{1}+\alpha_{2} z_{2}\right)+f$, and suppose $w \in C(0, \infty)$ is such that $w>0, w^{\prime} \geqslant 0$, and the integrals $\int^{\infty} w g z_{i} d t \quad(i=1,2)$ converge. Suppose also that $\int^{\infty}|b|\left(\left|z_{1}\right|+\left|z_{2}\right|\right)^{2} d t<\infty$. Then, applying Theorem 3 with $W=w I$ to (45) shows that (43) has a solution which satisfies (44), with

$$
x_{i}(t)=\alpha_{i}+o(1 / w(t)), \quad i=1,2 .
$$

The special case of this with $w=1$ and $f=0$ is known [5].

EXAMPLE 5. If (42) is nonoscillatory, we may choose $z_{1}$ and $z_{2}$ so that $z_{2} / z_{1}$ tends monotonically to infinity [3, p. 357]. Then it is useful to apply Theorem 3 to (45) with

$$
W=\operatorname{diag}\left[\left(z_{2} / z_{1}\right)^{q},\left(z_{2} / z_{1}\right)^{q+1}\right] \quad(q \geqslant 0) .
$$

For example, if $a=-1$ in (42), so that (43) becomes

$$
y^{\prime \prime}-y=b(t) y+f(t), \quad t>0,
$$

we take $z_{1}=e^{-t}$ and $z_{2}=e^{t}$ in (44) and (45), and

$$
W=\operatorname{diag}\left[e^{2 q t}, e^{2(q+1) t}\right] \quad(q \geqslant 0) .
$$

Theorem 3 then implies that if $\int^{\infty}|b(t)| d t<\infty$ and $\alpha_{1}$ and $\alpha_{2}$ are constants such that

$$
\int^{\infty} e^{2 q t}\left[b(t)\left(\alpha_{1}+\alpha_{2} e^{2 t}\right)+f(t) e^{t}\right] d t
$$

converges, then (46) has a solution $y$ such that

$$
y^{(r)}(t)=(-1)^{r} \alpha_{1} e^{-t}+\alpha_{2} e^{t}+o\left(e^{-(2 q+1) t}\right), \quad r=0,1 .
$$

This implies several results previously obtained under stronger integral conditions. 


\section{BIBLIOGRAPHY}

1. F. Brauer, Asymptotic equivalence and asymptotic behavior of linear systems, Michigan Math. J. 9 (1962), 33-43.

2. F. Brauer and J. S. W. Wong, On asymptotic hehavior of perturhed linear systems, J. Differential Equations 6 (1969), 142-153.

3. P. Hartman, Ordinary differential equations, Wiley, New York, 1964.

4. N. Onuchic, Nonlinear perturbations of a linear system of ordinary differential equations, Michigan Math. J. 11 (1964), 237-242.

5. W. F. Trench, On the asymptotic behavior of solutions of second order linear differential equations, Proc. Amer. Math. Soc. 14 (1963), 12-14.

6. Asymptotic hehavior of solutions of $L u=g\left(t, u, \ldots, u^{(k-1)}\right)$. J. Differential Equations 11 (1972), 38-48.

Department of Mathematical Sciences, Drexel University, Philadelphia, Pennsylvania 19104 